\title{
A Case of Intestinal Microsporidiosis in a Renal Transplant Recipient
}

\author{
Nidhi Doshi ${ }^{\mathrm{a}, \mathrm{d}}$, Zaw Thet ${ }^{\mathrm{b}}$, Thin Han ${ }^{\mathrm{b}, \mathrm{c}}$, Julieanne Martin ${ }^{\mathrm{b}}$
}

\begin{abstract}
Post-renal transplant diarrhea is a common clinical presentation. An extensive list of potential etiology adds to the diagnostic dilemma. In cases of prolonged or intractable diarrhea, invasive tests are often performed. Intestinal microsporidia can be diagnosed with simple non-invasive stool polymerase chain reaction (PCR). Based on this case, we propose an easy to follow flow chart and present a literature review on post-renal transplant diarrhea. Further multicenter validation testing is required for the proposed flow chart.
\end{abstract}

Keywords: Microsporidiosis; Renal transplant recipient; Diarrhea

\section{Introduction}

This is a unique case which was managed in a regional hospital in Australia. Diagnosis of microsporidiosis is often delayed. A series of investigations are undertaken before the diagnosis is reached. Often the cause of diarrhea is empirically presumed to be immunosuppressants. Modification of immunosuppressant has risk of resulting in rejection of the transplant.

In literature it has been cited that the onset of symptom occurs within 6 to 12 months post-transplant, but in our case it occurred 6 years after the transplant. Microsporidiosis is still under-recognized resulting in delayed diagnosis and increased morbidity through heightened risk of transplant rejection due to immunosuppression being adjusted. Non-invasive stool polymerase chain reaction (PCR) test should be considered early in the diagnostic workup. Diarrhea is a very common presentation in solid organ transplant (SOT), despite that there is no

Manuscript submitted July 20, 2019, accepted July 30, 2019

aDepartment of General Medicine, Gold Coast University Hospital, QLD, Australia

bDepartment of Nephrology, Central Queensland Hospital and Health Service, Rockhampton, QLD, Australia

'Rural Clinical School, University of Queensland, Rockhampton, QLD, Australia

${ }^{\mathrm{d} C}$ Corresponding Author: Nidhi Doshi, Department of General Medicine, Gold Coast University Hospital, 34 Buccaneer Way, Coomera, QLD 4209, Australia. Email: drnids@yahoo.co.uk

doi: https://doi.org/10.14740/jmc3340 universally agreed or region-specific guidance. We have come up with a unique flow chart that is easy to understand, can be cost effective and reduce morbidity. We suggest that validity of the flow chart be tested in larger multicenter studies in future.

\section{Case Report}

A 58-year-old man received a deceased donor renal transplant in 2014. The cause for his end-stage renal failure was unknown. Prior to renal transplant, he had 6 years of hemodialysis. Post-transplant, he was prescribed standard immunosuppressive therapy including tacrolimus, mycophenolate mofetil and prednisolone. His other comorbidities were hypertension treated with ramipril and gout that was well controlled with allopurinol. He was of Caucasian origin and worked as a tradesman.

Three years post-transplant, he presented to the outpatient renal clinic with symptoms of diarrhea for 2 weeks. He was afebrile and had no abdominal pain. Stool was described as watery with a frequency of about 10 to 14 times per day. There was no mucus or blood present in the stool. On clinical examination, he was tachycardic to 110 per min and blood pressure was $92 / 68$ $\mathrm{mm} \mathrm{Hg}$. His tongue was moderately dry. His abdomen was soft and non-tender. Respiratory and cardiac examination was unremarkable. He was admitted for initial investigations and fluid resuscitation. His inflammatory markers were normal and renal function showed urea of $8.3 \mathrm{mmol} / \mathrm{L}$, creatinine $138 \mu \mathrm{mol} / \mathrm{L}$ and glomerular filtration rate (GFR) $49 \mathrm{ml} / \mathrm{min}$ (baseline GFR 60 - 62). Potassium level was $3.4 \mathrm{mmol} / \mathrm{L}$. Tacrolimus drug level was within the target range. Fecal assay was negative for routine bacterial, viral and protozoan pathogens.

The dose of mycophenolate mofetil was split from $720 \mathrm{mg}$ $\mathrm{BD}$ to $360 \mathrm{mg}$ QID. Following improvement in his symptoms and return of renal function to baseline, he was discharged home.

At outpatient review 2 weeks post-discharge, he described persistent symptoms. His stool frequency had increased to 16 per day. He had maintained his hydration level with increased fluid intake. He was hemodynamically stable. To expedite further investigations, he was re-admitted and referred for inpatient colonoscopy and biopsy. Biopsy result showed parasitophorous vesicles containing finely granular eosinophilic structures, suspicious for microsporidia. Subsequent test for microsporidia on stool PCR was positive.

After nearly 6 weeks of investigations and invasive tests, 
Table 1. Differential Diagnosis of Diarrhea in Renal Transplant Recipients

\author{
Drug related \\ Mycophenolate, tacrolimus, antibiotics, proton pump inhibitors, metformin \\ Infective causes \\ Bacterial: salmonella, C. difficile, campylobacter, shigella, yersinia \\ Viral: $\mathrm{CMV}$, norovirus and rotavirus \\ Parasitic: cryptosporidium, giardia, entamoeba, microsporidia \\ Surgical causes \\ Intra-abdominal sepsis, mesenteric ischemia and colorectal carcinoma \\ Miscellaneous \\ Coeliac disease, irritable bowel syndrome, malabsorption syndrome, post-transplant lymphoproliferative disorder
}

CMV: cytomegalovirus.

he was diagnosed with intestinal microsporidiosis. Subsequently, he was commenced on albendazole $400 \mathrm{mg}$ twice daily. Within $48 \mathrm{~h}$ of albendazole treatment, stool frequency reduced to six per day. He was observed for 1 more day and then was discharged home.

On follow-up phone call at 7-day post-discharge, he described stool consistency as formed and improvement in frequency of bowel motion to two per day. At further 6-week follow-up, he was symptom-free. Repeat test for microsporidia was negative on stool PCR. Albendazole was stopped at the end of 4 weeks of therapy.

\section{Literature Review and Discussion}

World Health Organization defines diarrhea as three or more loose stools per day and persistent diarrhea as symptoms of more than 14-day duration [1]. Symptoms lasting longer than a month are defined as chronic. Diarrhea is a frequent complication after renal transplant. The incidence of diarrhea is highest in the first-year post-renal transplant. It has been estimated that one in 10 patients are affected with diarrhea in the first year post-renal transplant [2-4].

Acute diarrhea in a post-renal transplant recipient has twofold risk of graft rejection due to variable absorption of immunosuppressants. In post-renal transplant diarrhea (PRTD), there is reduction in p-glycoprotein enzyme activity with a resultant higher tacrolimus level and potential subsequent renal toxicity [2]. Chronic diarrhea can result in hyperoxaluria, which causes inflammation and graft rejection [2].
In renal transplant patients, up to $64 \%$ of diarrheal cases are thought to be infectious in origin [2]. Clostridium difficile (C. difficile), cytomegalovirus (CMV) and norovirus are the commonest pathogens in PRTD [3]. However, diarrhea may also be due to factors not related to immunosuppressive therapy such as oral hypoglycemic agents, broad-spectrum antibiotics or inflammatory bowel disease (Tables 1 and 2) [4, 5].

Microsporidia have been known to cause infection in HIV patients [6-8]. Over the last 20 years, 31 cases of intestinal and disseminated microsporidiosis have been cited in renal transplant recipients [9-14]. It has been estimated that microsporidia accounts for approximately $3.5 \%$ of all post-transplant diarrhea $[12,13]$. In 2012, George et al reported the first case of disseminated microsporidiosis in a non-HIV renal transplant recipient in Australia [8]. In the last decade, microsporidia has been recognized as the emerging opportunistic pathogen that causes diarrhea in a SOT recipient as well as in other immunosuppressed patients such as those who have diabetes, hematological malignancy, rheumatological conditions receiving anti-tumor necrosis treatment, in elderly and in patients who are on long-term corticosteroids [13, 14].

Microsporidia are a group of obligate intracellular parasites [15]. Fourteen species have been identified that cause diarrhea in humans $[8,15]$. Amongst the microsporidia species, Enterocytozoon bieneusi (E. bieneusi), cuniculi, intestinalis and hellen are the commonest human pathogens [16-18].

Transmission of spores occurs through feco-oral route from infected humans, animals or contaminated food and water. It has been cited that preceding high-dose corticosteroid treatment, concurrent CMV and giardia infection can predis-

Table 2. Diagnostic Investigations for Diarrhea in a Renal Transplant Recipient

Non-invasive

Stool: PCR, microscopy

Blood (serum): blood culture, CMV PCR and quantitative assay, immunosuppressive drug serum assays, immunological test for endomysial antibodies and transglutamase antibodies

Invasive

Biopsy: colonic, terminal ileum, gastric, duodenal 


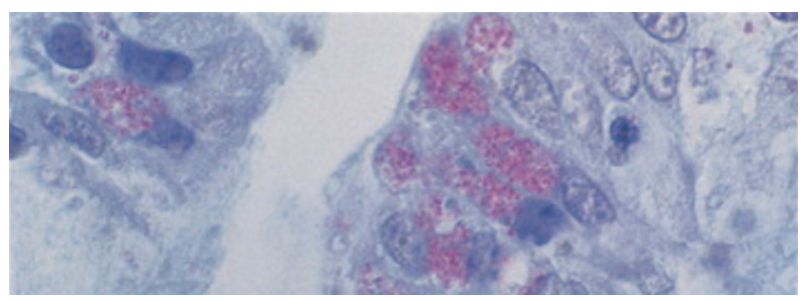

Figure 1. Jejunal biopsy containing microsporidia in an enterocyte.

pose to microsporidia infection [9]. In 2015, Hocevar et al reported cluster of three microsporidia cases in SOT recipients, acquired through a common infected donor [19].

The onset of symptoms varies from 6 to 60 weeks posttransplant; however, the majority present within 6 to 12 months. The symptoms usually last from 1 to 40 weeks, with a median period of 2 weeks [14]. In an immunocompromised host, microsporidia primarily infect enterocytes, though several case reports of disseminated disease involving respiratory, renal, central nervous systems and eyes have been described [8, 20-25].

Several laboratory tests are available for identification of spores and confirmation of infection [26]. Traditional methods such as light microscopy using modified trichrome stains or Giemsa (special stains) are inexpensive methods but lack species identification. Serological tests using enzyme-linked immunosorbent assay (ELISA) lack sensitivity in differentiating recent versus past infection and therefore are not recommended for routine diagnostic use [27]. Immunofluorescent techniques using reagents are only available in research laboratories. Spores can be detected in centrifuged specimen from duodenal aspirate, colony-stimulating factor (CSF), bronchoalveolar lavage and bile. In disseminated microsporidiosis, renal involvement is relatively common and testing of urine specimen for spores is recommended [26, 27].

The molecular diagnostic tests using PCR detects microsporidia in stool, urine and various biopsy specimens [2]. In comparison with light microscopy, molecular testing is more sensitive and provides additional benefit of species identification. This information has therapeutic advantage in initiating species-specific treatment $[23,28]$.

In 2013, Coste et al studied severe diarrhea in 49 renal transplant recipients [4]. Detection rate of pathogens was only $23 \%$ using classic microbiological methods, whereas using multiplex PCR the detection of pathogens increased to $72 \%$ [2]. In clinical practice routine tests are often negative, leading to modification of immunosuppressive medications $[2,5,29]$.

Identifying microsporidia on a routine hematoxylin and eosin stain can be challenging and often required an experienced pathologist. Intestinal infection can be patchy and missed on the biopsies. In biopsy specimen, microsporidia are seen within enterocytes and in lamina propria (Fig. 1).

Other histological features include minute ulcer over the duodenal and colonic folds and a necrotic patch over the ulcers [30].

A superficial biopsy may only reveal the necrotic cells. Deeper tissue biopsy carries the risk of perforation. Body fluids and stool examination are equally sensitive, and these

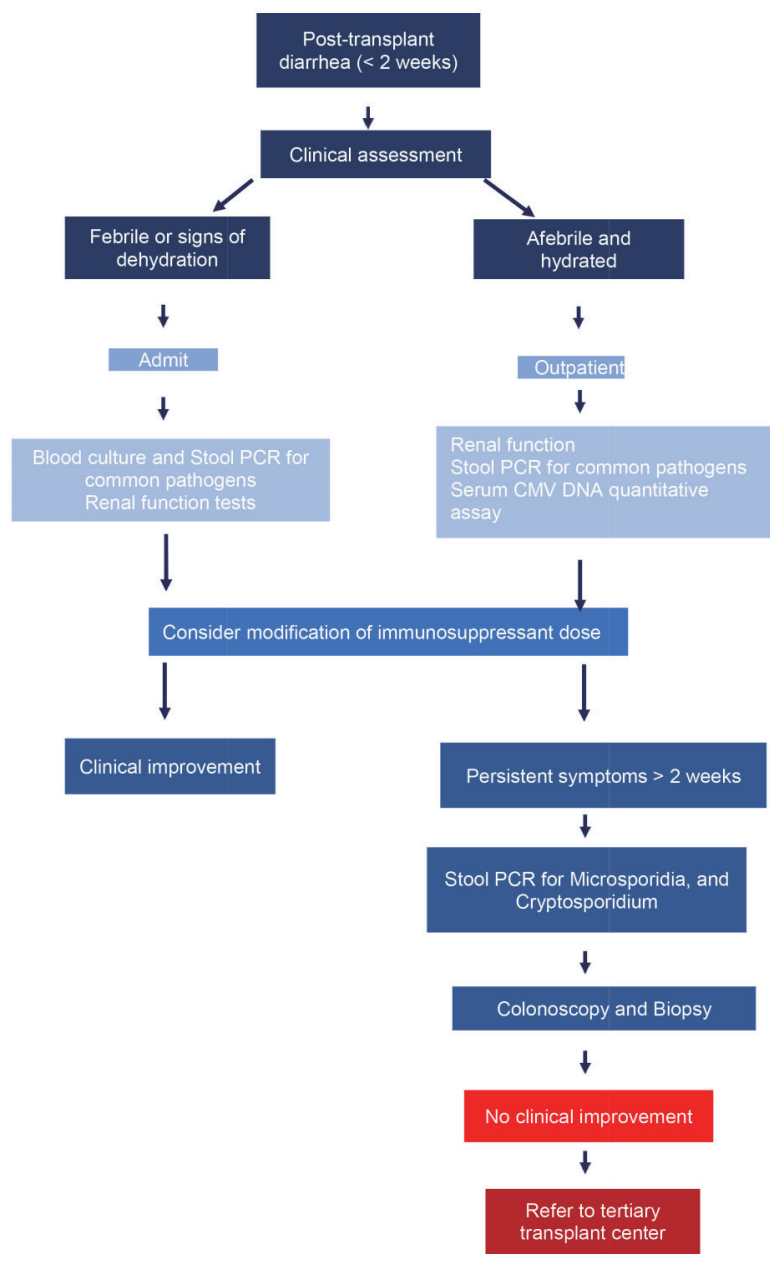

Figure 2. Proposed diagnostic algorithm for diarrhea in a renal transplant recipient.

specimens can be repeatedly collected based on clinical presentation. Most authors recommend biopsy as the second-line investigation; however, the yield is no more sensitive than stool and body fluid examination $[18,31]$.

Often negative initial investigation leads nephrologists to perform invasive tests such as colonoscopy [2]. Modification of immunosuppressive treatment, considered as the cause of diarrhea, has risk of acute or chronic transplant rejection [1, $2,25]$.

With stepwise testing strategy, costs can be reduced without compromising diagnostic yields. In the first-stage testing, authors recommend assessment for $C$. difficile, food-borne bacterial and viral pathogens (Fig. 2). For persistent diarrhea, second-stage evaluation should include stool PCR for giardia, cryptosporidium, microsporidia and possibly colonoscopy. In a landmark study it was shown that diarrhea resolved in approximately $50 \%$ patients without modification of immunosuppressant drugs [29].

As susceptibility of microsporidia species to different drugs is variable, species identification is recommended for appropriate treatment $[32,33]$. Albendazole is efficacious in treating E. intestinalis but less effective against E. bieneusi [4]. 
Table 3. Summary of Literature Review on Efficacy of Albendazole in Microsporidiosis

\begin{tabular}{|c|c|c|c|}
\hline Article/reference & Intestinal/disseminated infection & Treatment agent/dose/duration & Outcome \\
\hline Dacha et al [34] & Intestinal E. intestinalis & Albendazole $400 \mathrm{mg}$ BD for 4 weeks & Effective \\
\hline Galvan et al [14], patient 1 & Intestinal & $\begin{array}{l}\text { Initially metronidazole, on relapse switched } \\
\text { to albendazole } 400 \mathrm{mg} \text { BD for } 3 \text { weeks }\end{array}$ & Albendazole effective \\
\hline Galvan et al [14], patient 2 & Intestinal & Immunosuppression withdrawal & \\
\hline George et al [8] & Disseminated & Albendazole $400 \mathrm{mg} \mathrm{BD}$, duration not defined & Effective \\
\hline
\end{tabular}

Fumagillin, an antibiotic derived from the fungus Aspergillus fumigatus, is often efficacious against $E$. bieneusi. Fumagillin use is limited by severe toxicity mainly bone marrow suppression and thrombocytopenia [34, 35].

Two weeks of albendazole treatment is associated with increased risk of relapse. Consequently, in this case, treatment was continued for a total of 4 weeks. To prevent relapses, it is recommended that microbiological clearance should be the end-point of treatment [12, 36-38] (Table 3) [8, 14, 26, 34].

Although species identification was not performed in this case, given the clinical and microbiological response, we believe the infection was likely due to E. intestinalis.

In summary, post-transplant diarrhea is associated with high morbidity and mortality. Transplant patients should be considered as high-risk group for microsporidiosis. In absence of common pathogens on initial investigations or poor response to standard treatment, microsporidiosis should be considered in the list of differential diagnosis.

\section{Conclusions}

Microsporidiosis is an emerging disease in non-HIV SOT recipients. Investigation for microsporidiosis should be considered in diagnostic workup for renal transplant patients who present with persistent diarrhea. Authors propose a flow chart for management of diarrhea with a focus on microsporidiosis as an early investigation. This flow chart will require validation in a future multicentre study.

\section{Acknowledgments}

None to declare.

\section{Financial Disclosure}

None to declare.

\section{Conflict of Interest}

None to declare.

\section{Informed Consent}

Informed consent to publish this case report was taken from the patient.

\section{Author Contributions}

ND is primary author, who was involved in the literature review and final proofreading; ZT and TH contributed to the case report; JM contributed to correction and proofreading.

\section{References}

1. WHO definition of persistent diarrhoea. May 2017. Accessed on 15/03/2019. Available on: https://www.who. int/news-room/fact- sheets/detail/diarrhoeal-disease.

2. Florence Aulagnon, et al. Diarrhoea after kidney transplantation: a new look at a frequent symptom. Transplantation. 2014;98(8):806-816.

3. Echenique IA, Penugonda S, Stosor V, Ison MG, Angarone MP. Diagnostic yields in solid organ transplant recipients admitted with diarrhea. Clin Infect Dis. 2015;60(5):729737.

4. Coste JF, Vuiblet V, Moustapha B, Bouin A, Lavaud S, Toupance $\mathrm{O}$, de Rougemont A, et al. Microbiological diagnosis of severe diarrhea in kidney transplant recipients by use of multiplex PCR assays. J Clin Microbiol. 2013;51(6):1841-1849.

5. Maes B, Hadaya K, de Moor B, Cambier P, Peeters P, de Meester J, Donck J, et al. Severe diarrhea in renal transplant patients: results of the DIDACT study. Am J Transplant. 2006;6(6):1466-1472.

6. Kotton CN. Life-saving organ transplants accompanied by stealthy and unexpected pathogens. Ann Intern Med. 2014;160(4):282-283.

7. Nagpal A, Pritt BS, Lorenz EC, Amer H, Nasr SH, Cornell LD, Iqbal S, et al. Disseminated microsporidiosis in a renal transplant recipient: case report and review of the literature. Transpl Infect Dis. 2013;15(5):526-532.

8. George B, Coates T, McDonald S, Russ G, Cherian S, Nolan J, Brealey J. Disseminated microsporidiosis with Encephalitozoon species in a renal transplant recipient. 
Nephrology (Carlton). 2012;17(Suppl 1):5-8.

9. Ghoshal U, Khanduja S, Pant P, Prasad KN, Dhole TN, Sharma RK, Ghoshal UC. Intestinal microsporidiosis in renal transplant recipients: Prevalence, predictors of occurrence and genetic characterization. Indian J Med Microbiol. 2015;33(3):357-363.

10. Brown M, Longano A, Dendle C, Polkinghorne KR, Kanellis J. Confirmed microsporidial graft infection in a HIV-negative renal transplant recipient: A case report and review of the literature. Transpl Infect Dis. 2018;20(3):e12888.

11. Leder K, Ryan N, Spelman D, Crowe SM. Microsporidial disease in HIV-infected patients: a report of 42 patients and review of the literature. Scand J Infect Dis. 1998;30(4):331-338.

12. Lanternier F, Boutboul D, Menotti J, Chandesris MO, Sarfati C, Mamzer Bruneel MF, Calmus Y, et al. Microsporidiosis in solid organ transplant recipients: two Enterocytozoon bieneusi cases and review. Transpl Infect Dis. 2009;11(1):83-88.

13. Metge S, Van Nhieu JT, Dahmane D, Grimbert P, Foulet F, Sarfati C, Bretagne S. A case of Enterocytozoon bieneusi infection in an HIV-negative renal transplant recipient. Eur J Clin Microbiol Infect Dis. 2000;19(3):221-223.

14. Galvan AL, Sanchez AM, Valentin MA, Henriques-Gil N, Izquierdo F, Fenoy S, del Aguila C. First cases of microsporidiosis in transplant recipients in Spain and review of the literature. J Clin Microbiol. 2011;49(4):1301-1306.

15. Centre for disease control and prevention. Microsporidiosis. Last updated on 29th May 2017. Available on: https:// www.cdc.gov/dpdx/microsporidiosis/index.html.

16. Kotler DP, Orenstein JM. Clinical syndromes associated with microsporidiosis. Adv Parasitol. 1998;40:321-349.

17. Teachey DT, Russo P, Orenstein JM, Didier ES, Bowers C, Bunin N. Pulmonary infection with microsporidia after allogeneic bone marrow transplantation. Bone Marrow Transplant. 2004;33(3):299-302.

18. Kicia M, Wesolowska M, Kopacz Z, Jakuszko K, Sak B, Kvetonova D, Krajewska M, et al. Prevalence and molecular characteristics of urinary and intestinal microsporidia infections in renal transplant recipients. Clin Microbiol Infect. 2016;22(5):462 e465-469.

19. Hocevar SN, Paddock CD, Spak CW, Rosenblatt R, DiazLuna H, Castillo I, Luna S, et al. Microsporidiosis acquired through solid organ transplantation: a public health investigation. Ann Intern Med. 2014;160(4):213-220.

20. Hernandez-Rodriguez OX, Alvarez-Torres O, Ofelia Uribe-Uribe N. Microsporidia infection in a mexican kidney transplant recipient. Case Rep Nephrol. 2012;2012:928083.

21. Kakrania R, Joseph J, Vaddavalli PK, Gangopadhyay N, Sharma S. Microsporidia keratoconjunctivitis in a corneal graft. Eye (Lond). 2006;20(11):1314-1315.

22. Didier ES, Weiss LM. Microsporidiosis: not just in AIDS patients. Curr Opin Infect Dis. 2011;24(5):490-495.

23. Carlson JR, Li L, Helton CL, Munn RJ, Wasson K, Perez $\mathrm{RV}$, Gallay BJ, et al. Disseminated microsporidiosis in a pancreas/kidney transplant recipient. Arch Pathol Lab Med. 2004;128(3):e41-43.
24. Kicia M, Wesolowska M, Jakuszko K, Kopacz Z, Sak B, Kvetonova D, Krajewska M, et al. Concurrent infection of the urinary tract with Encephalitozoon cuniculi and Enterocytozoon bieneusi in a renal transplant recipient. J Clin Microbiol. 2014;52(5):1780-1782.

25. Talabani H, Sarfati C, Pillebout E, van Gool T, Derouin F, Menotti J. Disseminated infection with a new genovar of Encephalitozoon cuniculi in a renal transplant recipient. J Clin Microbiol. 2010;48(7):2651-2653.

26. Gamboa-Dominguez A, De Anda J, Donis J, Ruiz-Maza F, Visvesvara GS, Diliz H. Disseminated encephalitozoon cuniculi infection in a Mexican kidney transplant recipient. Transplantation. 2003;75(11):1898-1900.

27. Garcia LS. Laboratory identification of the microsporidia. J Clin Microbiol. 2002;40(6):1892-1901.

28. Orenstein JM, Russo P, Didier ES, Bowers C, Bunin N, Teachey DT. Fatal pulmonary microsporidiosis due to encephalitozoon cuniculi following allogeneic bone marrow transplantation for acute myelogenous leukemia. Ultrastruct Pathol. 2005;29(3-4):269-276.

29. Goetz M, Eichenlaub S, Pape GR, Hoffmann RM. Chronic diarrhea as a result of intestinal microsposidiosis in a liver transplant recipient. Transplantation. 2001;71(2):334337.

30. Khanduja S, Ghoshal U, Ghoshal UC. Phylogenetic analysis of genetically distinct Enterocytozoon bieneusi infecting renal transplant recipients. Acta Parasitol. 2017;62(1):63-68.

31. Mahmood MN, Keohane ME, Burd EM. Pathologic quiz case: a 45-year-old renal transplant recipient with persistent fever. Arch Pathol Lab Med. 2003;127(4):e224-226.

32. Sing A, Tybus K, Heesemann J, Mathis A. Molecular diagnosis of an Enterocytozoon bieneusi human genotype $\mathrm{C}$ infection in a moderately immunosuppressed human immunodeficiency virus seronegative liver-transplant recipient with severe chronic diarrhea. J Clin Microbiol. 2001;39(6):2371-2372.

33. Serra S, Jani PA. An approach to duodenal biopsies. J Clin Pathol. 2006;59(11):1133-1150.

34. Dacha S, Pham D, Cai Q. Microsporidiosis: an unusual endoscopic finding. Clin Gastroenterol Hepatol. 2014;12(5):e37-38.

35. Guerard A, Rabodonirina M, Cotte L, Liguory O, Piens MA, Daoud S, Picot S, et al. Intestinal microsporidiosis occurring in two renal transplant recipients treated with mycophenolate mofetil. Transplantation. 1999;68(5):699707.

36. Audemard A, Le Bellec ML, Carluer L, Dargere S, Verdon R, Castrale C, Lobbedez T, et al. Fumagillininduced aseptic meningoencephalitis in a kidney transplant recipient with microsporidiosis. Transpl Infect Dis. 2012;14(6):E147-149.

37. Champion L, Durrbach A, Lang P, Delahousse M, Chauvet C, Sarfati C, Glotz D, et al. Fumagillin for treatment of intestinal microsporidiosis in renal transplant recipients. Am J Transplant. 2010;10(8):1925-1930.

38. Weiss LM. Microsporidiosis. Mandell, Douglas and Bennett's principles and practice of infectious diseases (Eighth Edition). 2015. 